\title{
Challenging Dogmas in IBD
}

The field of IBD is rapidly evolving. Our knowledge of genetic predisposition, immunological mechanisms and disease behavior has never expanded so widely as in the last decade, with currently $2-4$ new scientific papers on IBD published per day. Our tools to accurately visualize the extent and severity of inflammation and to detect dysplasia and cancer have become highly reliable. The list of novel small molecules and biologic agents targeting various mechanisms in the inflammatory cascade and in the vascular adhesion system is virtually endless.

Yet many relevant questions remain unanswered when it comes to daily IBD management. What is the optimal target for disease control? Why does IBD often 'escape' after a period of successful response to certain treatments? Why does the disease behavior sometimes change drastically during the disease course? Why is postoperative recurrence of Crohn's disease virtually inevitable?

Although we are convinced that we had found some answers to these questions, we need to realize that many such 'dogmas' need to be questioned and readdressed. We really need to make more effort to get to the 'heart of the matter'.

This issue has given an overview of most of the presentations at the 106th FALK symposium in Brussels which tried to contribute to a critical attitude towards 'ancient beliefs' in the field of IBD. The era of evidence-based medicine forces us to critically review scientific data, to rephrase certain standards and, above all, to translate novel insights into daily care for our patients.

The authors certainly did a magnificent job in writing their chapters. They offer an exciting scientific and clinical journey through the world of IBD and will hopefully give you a clearer view of the intriguing enigma that is IBD.

Geert D'Haens, $M D$, PhD, Amsterdam

Also on behalf of Severine Vermeire and Axel Dignass 